\title{
Geometric Reformulation of 3-Fingered Force-Closure Condition
}

\author{
Attawith Sudsang and Thanathorn Phoka \\ Department of Computer Engineering \\ Chulalongkorn University \\ Bangkok 10330, Thailand \\ \{attawith,phoka\}@cp.eng.chula.ac.th
}

\begin{abstract}
This paper addresses the problem of testing whether three contact points form a 3-fingered force-closure grasp in two dimensions. In particular, assuming frictional point contacts, we present a new necessary and sufficient condition for three fingers to form a force-closure grasp. The proposed condition is based on a technique for representing a friction cone as a line segment in a dual plane. This representation allows force-closure test to be formulated as the problem of intersection detection between a line segment and a convex polygon. The resulting geometric condition is presented along with an efficient algorithm for using the condition in force-closure test.
\end{abstract}

\section{INTRODUCTION}

In robotics grasping, it is desirable that the hand can hold the object securely amidst any external disturbances. This intuitive quality of a stable grasp is the root of a classical concept in grasping known as the force-closure property [1], [7]. A grasp is said to achieve force-closure when any external wrench can be balanced by wrenches at the fingertips. This paper addresses the problem of testing whether three contact points form a 3-fingered force-closure grasp in two dimensions. In particular, assuming frictional point contacts, we present a new necessary and sufficient condition for three fingers to form a force-closure grasp. The proposed condition is based on a technique for representing a friction cone as a line segment in a dual plane. This representation allows force-closure test to be formulated as the problem of intersection detection between a line segment and a convex polygon.

Force-closure test is a basic problem in grasping. The notion of force-closure however does not directly yield a method for force-closure test. Some necessary and sufficient conditions for force-closure grasps were formulated in order to derive such a test. Different conditions usually result in different methods varying in efficiency and applicability. A widely used necessary and sufficient force-closure condition given by Salisbury and Roth [7] allowed a force-closure test to be performed by checking whether the origin is strictly inside the convex hull of the primitive contact wrenches. This test also provided an underlying idea to recent work in grasp computation [3]. In [5], Nguyen formally demonstrated for 2-fingered grasps that nonmarginal equilibrium grasps achieve force-closure. He also gave a simple geometric algorithm for 2-fingered force-closure grasp planning. His work was extended to the cases of 3 fingers by Ponce and Faverjon [6]. In the paper, they proposed a 3fingered grasp planning method for polygonal objects based on linear programming. The approach directly followed one of their sufficient condition for force-closure grasps. Recently, Jia-Wei Li et al. [4] investigated a necessary and sufficient condition for 3-fingered force-closure grasps from [6] and developed an algorithm for 3-fingered force-closure test. Their method begins by preprocessing the friction cones using an operation called disposition. This operation properly shrinks the cones so that force-closure test can be reduced to detecting intersection of the three shrunk cones. Our method provides an alternative to their approach. Instead of actually modifying the cones, each cone is mapped to a line segment in the dual plane. Since properties of friction cones are preserved under this transformation, besides yielding an efficient force-closure test, our dual representation provides a new way for studying problems involving the force-closure property.

The rest of the paper is organized as follows. In Section II, background about force-closure condition is briefly reviewed. Most importantly, we recall Proposition 2 for which the proposed condition is based on. In Section III, we present the underlying geometry of cones that allows a double-sided friction cone to be represented as a line segment in a dual plane. This representation plays a crucial role in the derivation of the proposed condition. The main result is then given in Section IV where the proposed necessary and sufficient forceclosure condition is stated in Proposition 3. Minor limitation of this proposition and a workaround is discussed in Section $\mathrm{V}$. We finally conclude the paper with some future works in Section VI.

\section{BACKGROUND}

In two dimensions, a hard finger in contact with some object at a point $\boldsymbol{x}=\left(x_{1}, x_{2}\right)$ exerts a force $\boldsymbol{f}=\left(f_{1}, f_{2}\right)$ with moment $\operatorname{det}(\boldsymbol{x}, \boldsymbol{f})=x_{1} f_{2}-f_{1} x_{2}$ with respect to the origin (but it cannot exert a pure torque). Force and moment are combined into a three dimensional wrench $\boldsymbol{w}=(\boldsymbol{f}, \operatorname{det}(\boldsymbol{x}, \boldsymbol{f}))$. Under Coulomb friction, the set of wrenches that can be applied by the finger is:

$$
W=\{(\boldsymbol{f}, \operatorname{det}(\boldsymbol{x}, \boldsymbol{f})): \boldsymbol{f} \in C\},
$$


where $C$ denotes the friction cone at $\boldsymbol{x}$.

A $d$-finger grasp is defined geometrically by the position $\boldsymbol{x}_{i}(i=1, \ldots, d)$ of the fingers on the boundary of the grasped object. We can associate with each grasp the set of wrenches $W \subset \Re^{3}$ that can be exerted by the fingers. If we denote by $W_{i}$ the wrench set associated with the $i^{t h}$ finger, we have

$$
W=\left\{\sum_{i=1}^{d} \boldsymbol{w}_{i}: \boldsymbol{w}_{i} \in W_{i} \text { for } i=1, \ldots, d\right\} .
$$

Definition 1: A two dimensional grasp is said to achieve force closure when the corresponding wrench set $W$ is equal to $\Re^{3}$.

In other words, a grasp achieves force closure when any external wrench can be balanced by wrenches at the fingertips. A somewhat weaker condition is equilibrium, defined below.

Definition 2: A grasp is said to achieve equilibrium when there exist forces (not all of them being zero) in the friction cones at the fingertips such that the sum of the corresponding wrenches is zero.

Force-closure clearly implies equilibrium. More interestingly, it is formally shown in [5] for two finger cases and generalized to three finger cases in [6] that a sufficient condition for force closure is non-marginal equilibrium grasps, i.e., grasps such that the forces achieving equilibrium lie strictly inside the friction cones at the fingertips.

Proposition 1: A sufficient condition for 2- and 3-finger force closure is non-marginal equilibrium

That is, grasps achieving equilibrium with non-zero forces for some friction coefficient achieve force closure for any strictly greater friction coefficient. Due to [6], Proposition 2 below, which requires the following definition, characterizes 3-finger grasps achieving equilibrium with non-zero contact forces

Definition 3: A set of vectors positively spans $\Re^{n}$ if any vector in $\Re^{n}$ can be written as a positive linear combination of the set.

In $\Re^{2}$, a necessary and sufficient condition for three vectors to positively span the plane is obviously that they do not all lie in the same half plane.

Proposition 2: A necessary and sufficient condition for three points to form an equilibrium grasp with three non-zero contact forces, not all of them being parallel, is that $(\mathrm{Pa})$ there exist three lines in the corresponding double sided friction cones that intersect in a single point and $(\mathrm{Pb})$ the vectors parallel to these lines and lying in the internal friction cones at the contact points positively span the plane.

\section{GeOMETRY OF CONES}

Applying the force-closure condition given in Proposition 2 requires reasoning about forces in friction cones. Representation of forces and cones therefore plays an important role on how the condition can be tested or transformed. The objective of this section is to present geometry of cones that is the foundation of our new necessary and sufficient condition for force-closure grasps. Specifically, we present a representation technique that maps a cone in a primary plane into a line segment in the corresponding dual plane. This mapping is based on the well known duality of points and lines in two dimensions. It will become clear in the next section that being able to view a cone as a line segment is crucial to the formulation of the new condition.

The duality mapping discussed in this section is between the primary plane $(x, y)$ and the dual plane $(a, b)$. Friction cones are defined in the primary plane while the corresponding line segment will appear in the dual plane. Only lines that do not pass through the origin will be considered. Limitation caused by this assumption and a workaround will be examined in Section V.

Let us begin by recalling a basic duality mapping between points and lines in the plane. The coordinates $(x, y)$ of a point on a line that does not pass through the origin can be defined by an equation in the form $a x+b y=1$ where $a, b \in \Re$ dictate where the line intersects the $x$ and $y$ axes. This correspondence allows a line in the primary plane to be mapped to a point in the dual plane. More precisely, we have the following definition.

Definition 4: A line $a x+b y=1$ in the primary plane has a corresponding dual point $(a, b)$ in the dual plane.

With the duality relationship given above, it is obvious that the parameters $a$ and $b$ of every line in the primary plane that passes through the point $\left(x_{0}, y_{0}\right) \neq(0,0)$ must satisfy $a x_{0}+b y_{0}=1$ (Fig. 1). This equation essentially defines a line in the dual plane which, in turn, leads us to the following well known property of duality.
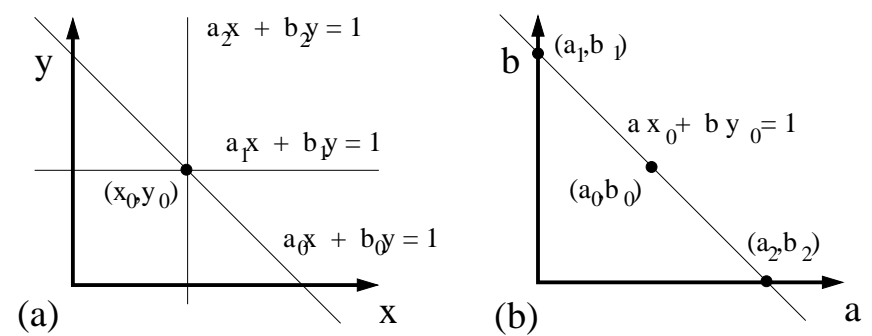

Fig. 1. Duality mapping: (a) three lines intersecting at a given point, and (b) the corresponding dual points of the three lines in the dual plane

Lemma 1: The dual points of all lines in the primary plane that intersect in a single point form a line in the dual plane.

As shown in Fig. 2(a), a double-sided cone can be considered as a set of all lines that pass through a common point (the cone's apex) such that they are entirely contained in the union of two opposing convex regions that are bounded by two crossing lines (the cone's boundary). By viewing a cone this way, it follows from Lemma 1 that the dual of a cone is a subset of a line in the dual plane. In fact, with little extra analysis, the following lemma can also be stated.

Lemma 2: If $D$ is a double-sided cone at the point $\boldsymbol{c}$ such that $D$ does not contain the origin and the angle $\phi$ from the 

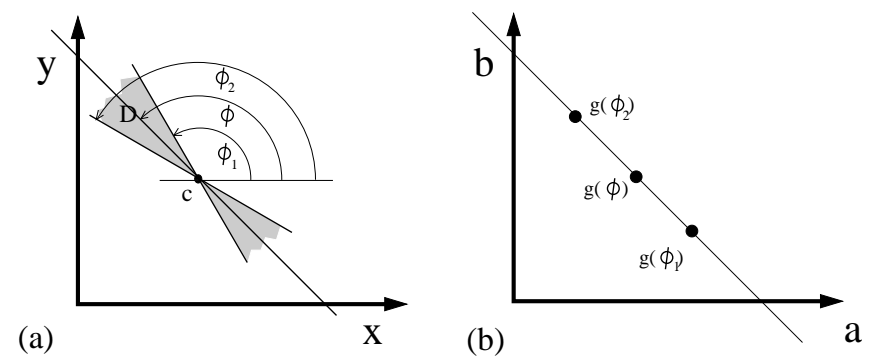

Fig. 2. Duality mapping of a cone: (a) a double-sided cone containing al lines through $c$ with orientations in the interval $\left[\phi_{1}, \phi_{2}\right]$, and (b) the dual poin of any line in the cone is between the two dual points of the two boundary lines of the cone

$+x$ axis to each line in $D$ is in the interval $\left[\phi_{1}, \phi_{2}\right]$, then the dual of $D$ in the dual plane is a line segment joining the point $\boldsymbol{g}\left(\phi_{1}\right)$ and $\boldsymbol{g}\left(\phi_{2}\right)$ where $\boldsymbol{g}: \Re \mapsto \Re^{2}$ is a vector function mapping angle $\phi$ to the dual point of a line whose angle from the $+x$ axis is $\phi$. (See Fig. 2)

Proof: To prove that the dual of the cone forms a line segment, it is sufficient to show that $\boldsymbol{g}$ is continuous on the interval $\left[\phi_{1}, \phi_{2}\right]$. Without loss of generality, let $\boldsymbol{g}(\phi)=(a(\phi), b(\phi))$ where $a(\phi) x+b(\phi) y=1$. To define the functions $a$ and $b$, let us consider the line passing through the point $\boldsymbol{c}=\left(x_{0}, y_{0}\right)$ and having $\phi$ as the angle from $+x$ axis to the line (Fig. 3). Clearly, this line can be defined by $\frac{y-y_{0}}{x-x_{0}}=\frac{\sin \phi}{\cos \phi}$. With some trigonometric simplification, this equation can be written in the form $a(\phi) x+b(\phi) y=1$ by having $a(\phi)=\frac{\sin \phi}{d \sin (\phi-\alpha)}$ and $b(\phi)=\frac{-\cos \phi}{d \sin (\phi-\alpha)}$ where $d=\sqrt{x_{0}^{2}+y_{0}^{2}}, x_{0}=d \cos \alpha$ and $y_{0}=d \sin \alpha$. This means that the functions $a$ and $b$ are continuous when the line does not pass through the origin. In turn, $\boldsymbol{g}$ is continuous in the interval $\left[\phi_{1}, \phi_{2}\right]$ as desired.

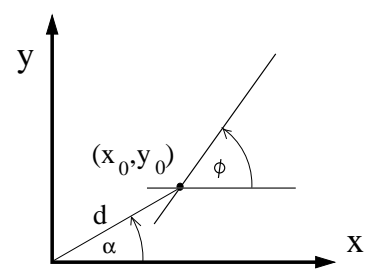

Fig. 3. A line not through the origin but through $\left(x_{0}, y_{0}\right)$ with the angle $\phi$ from the $+x$ axis

We also need to show that $\boldsymbol{g}\left(\phi_{1}\right)$ and $\boldsymbol{g}\left(\phi_{2}\right)$ are the endpoints of the line segment that is the dual of the cone. To do so, it is sufficient to show that $\boldsymbol{g}$ is one-to-one on the interval $\left[\phi_{1}, \phi_{2}\right]$. This is almost obvious. The line through $\boldsymbol{c}$ with angle $\phi$ from the $+x$ axis is identical only to the line through $c$ with angle $\phi+k \pi, k \in \mathcal{Z}$ from the $+x$ axis. Since $D$ is a cone, the interval $\left[\phi_{1}, \phi_{2}\right]$ must be smaller than $\pi$. This means that no two angles in the interval correspond to the same line. Together with the fact that different lines have different dual points, we can then conclude that $\boldsymbol{g}$ is one-to-one.

\section{New Force-Closure Condition}

To derive the proposed necessary and sufficient forceclosure condition, we transform Proposition 2 into a new one by totally rewriting the condition in the dual plane using the duality mapping given in Section III. The transformation can be best explained in two steps. In Section IV-A, we show how a key condition of Proposition 2 can be rewritten. Specifically, we state in Lemma 3 a condition in the dual plane for three forces to intersect in a single point and positively span the plane. Building upon this condition, Section IV-B develop a condition in the dual plane for checking whether there exist three forces in the three friction cones that satisfy Lemma 3. This later condition is essentially the proposed necessary and sufficient force-closure condition.

Application of forces is the most basic element to be considered in this section. In Proposition 2, when a contact force is involved, it is sufficient to pay attention only to the line of action and the direction of the force application along the line. When the force is not through the origin, it creates a moment around the origin. Without actually calculating the moment, the sign of moment (either positive or negative) can be obtained by inspecting the direction of the force around the origin. The counterclockwise direction gives a positive sign while the clockwise direction gives a negative one. This idea enables us to represent a contact force in the dual plane with the dual point of its line of action together with its sign of moment. This practice is used throughout this section.

\section{A. Concurrent and Positively Spanning Forces}

An important building block is given in the next lemma. In particular, it provides a condition in the dual plane for three forces to intersect in a single point and positively span the plane. Let us state the lemma and sketch how it can be proved.

Lemma 3: Three forces, none of them passing through the origin, intersect at a single point and positively span the plane if and only if $(\mathrm{Pa})$ the three dual points of the corresponding three lines of action (of the three forces) lie on the same line that does not contain the origin, and $(\mathrm{Pb})$ the sign of moment of the middle dual point is different from those of the other two.

Condition $(\mathrm{Pa})$ of the lemma follows directly from Lemma 1. The restriction that the line through the three dual points does not contain the origin is needed to exclude the case of three parallel forces. To show how condition $(\mathrm{Pb})$ is derived, consider three forces that intersect in a point $c$ such that none of them passes through the origin. Certainly, at least two of the forces must have the same sign of moment. Let us denote these two forces by $\boldsymbol{f}_{1}$ and $\boldsymbol{f}_{2}$, and denote the remaining force by $f_{3}$. Also, let us denote by $D$ the double-sided cone at $c$ that contains all lines going through $c$ and parallel to vectors $\alpha \boldsymbol{f}_{1}+\beta \boldsymbol{f}_{2}, \alpha, \beta \geq 0$. Since both $\boldsymbol{f}_{1}$ and $\boldsymbol{f}_{2}$ have the same sign of moment, it is easy to verify that the origin cannot be contained in $D$ (otherwise, the sign of moment of $f_{1}$ would be different from that of $f_{2}$ ). Using Lemma 2, the dual of 


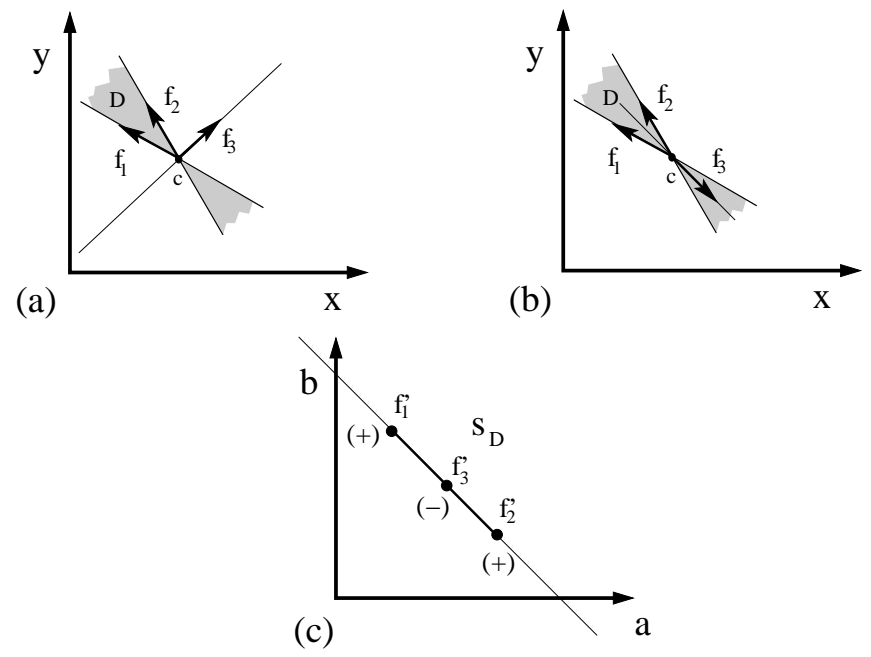

Fig. 4. Three intersecting forces: (a) not positively spanning the plane, (b) positively spanning the plane, and (c) the dual of the three forces that positively span the plane

$D$ is therefore a line segment whose endpoints are the dual points of the lines of action of $f_{1}$ and $f_{2}$. Let us call this line segment $S_{D}$. Now consider the remaining force $f_{3}$. For the three forces $f_{1}, f_{2}$ and $f_{3}$ to positively span the plane, it follows from Definition 3 that it is necessary and sufficient that they do not lie in the same half plane. As shown in Fig. 4(a), this implies that the line of action of $\boldsymbol{f}_{3}$ cannot be outside $D$ or the three forces would be pointing in the same half plane bounded by the line (regardless of the sign of moment of $\boldsymbol{f}_{3}$ ). The only remaining possibility is that the line of action of $\boldsymbol{f}_{3}$ must be contained in the interior of $D$ and the sign of moment of $f_{3}$ must be different from that of the other two forces (Fig. 4(b)). According to Lemma 2, the dual point of the line of action of $f_{3}$ is therefore in the interior of the line segment $S_{D}$ (Fig. 4(c)). With this conclusion and the fact that the sign of moment of $f_{3}$ is different from that of the other two forces, condition $(\mathrm{Pb})$ of the lemma follows.

\section{B. Formulating the Condition}

A friction cone is a cone of forces while the cone stated in Lemma 2 is a cone of lines. For convenience and accuracy of remaining discussion, the following definition is needed.

Definition 5: The underlying double-sided cone of a friction cone at a contact point is a double-sided cone at the same contact point that contains all lines of action of all forces in the friction cone.

Let $C_{i}, i=1,2,3$ be the three friction cones at three contact points $\boldsymbol{c}_{i}, i=1,2,3$ and assume that none of the forces in the three cones passes through the origin. Also, let us denote by $D_{i}$ the underlying double-sided cone of $C_{i}$. Since each $C_{i}$ is assumed to have no force that passes through the origin, the corresponding $D_{i}$ therefore does not contain the origin and, from Lemma 2, the dual of $D_{i}$ is a line segment in the dual plane, denoted $S_{i}$. When no force in a friction cone passes through the origin, it is obvious that all the forces create the same sense of rotation (either clockwise or counterclockwise) around the origin. In other words, every force in the same friction cone has the same sign of moment and, equivalently, every point on the same line segment $S_{i}$ in the dual plane has the same sign of moment as well (Fig. 5). We are now ready to state the main result. Let us give the proposed force-closure condition in the following proposition and explain how the condition is derived.
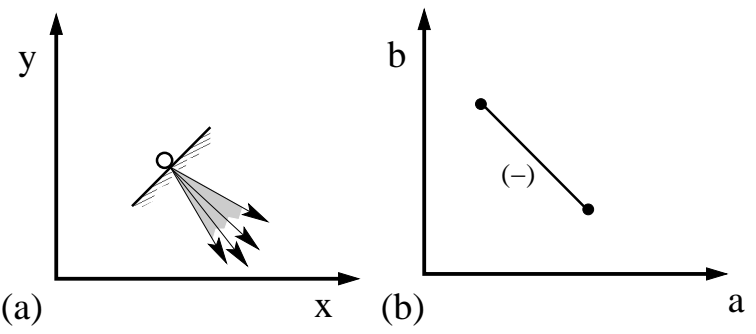

Fig. 5. A friction cone not containing the origin: (a) showing all forces in the cone, and (b) their dual line segment with the sign of moment

Proposition 3: Let $C_{i}, i=1,2,3$ be the three friction cones at three contact points such that none of the forces in the three cones passes through the origin. Also, let line segment $S_{i}$ be the dual of the underlying double-sided cone of $C_{i}$. A necessary and sufficient condition for the three contact points to form an equilibrium grasp with three non-zero contact forces is that (a) the three line segments $S_{i}, i=1,2,3$ do not have the same sign of moment, and (b) the convex hull of the two line segments with the same sign of moment intersect with the remaining line segment. See Fig. 6.

In essence, this proposition is an extension of Lemma 3. The proposition involves detecting whether there exist three forces, $f_{i} \in C_{i}, i=1,2,3$, or equivalently three dual points $\boldsymbol{p}_{i} \in S_{i}, i=1,2,3$, that satisfy Lemma 3 . Immediately from the condition $(\mathrm{Pb})$ of Lemma 3, we obtain the first condition of the proposition saying that the three dual line segments must not have the same sign of moment. Now, let $S_{k}$ and $S_{l}, k, l=1,2,3, k \neq l$, be the two dual line segments with the same sign of moment and let $S_{m}$ be the remaining dual line segment. For the dual points $\boldsymbol{p}_{k} \in S_{k}$ and $\boldsymbol{p}_{l} \in S_{l}$ such that the line segment $\overline{\boldsymbol{p}_{k} \boldsymbol{p}_{l}}$ intersects $S_{m}$, it is obvious that the three dual points $\boldsymbol{p}_{k}, \boldsymbol{p}_{l}$ and $\boldsymbol{p}_{m}=\overline{\boldsymbol{p}_{k} \boldsymbol{p}_{l}} \cap S_{m}$ satisfy Lemma 3. Since the union of all possible line segments $\overline{\boldsymbol{p}_{k} \boldsymbol{p}_{l}}$ is the convex hull of $S_{k}$ and $S_{l}$, it is obvious that there exists a line segment $\overline{\boldsymbol{p}_{k} \boldsymbol{p}_{l}}$ that intersects $S_{m}$ if and only if the convex hull intersects $S_{m}$. This, as a result, derives the second condition of the proposition.

\section{Algorithm}

With Proposition 3, testing whether three contact points can form a force-closure grasp is straightforward. We first apply Lemma 2 to convert each friction cone into the corresponding dual line segment. The two endpoints of a dual line segment 

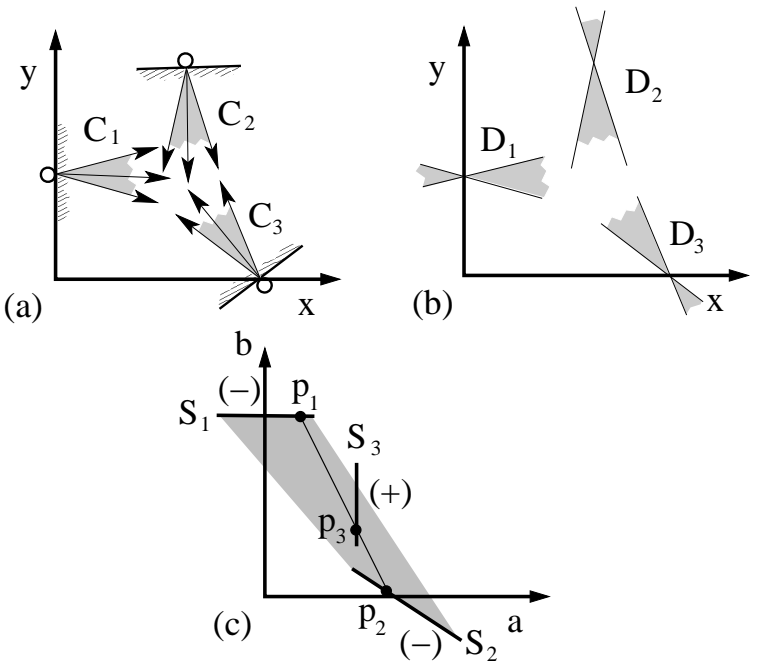

Fig. 6. Force-closure test: (a) three friction cones, (b) the underlying doublesided cone, and (c) their dual line segments showing the convex hull of the segments with the same sign of moment intersecting the other segment indicating force-closure

can be computed by applying the duality map given in Definition 4 to the two boundary lines of the corresponding friction cones. Of course, in some given configurations, the assumption of Lemma 2 may be violated because the origin is contained in some friction cones. In this case, a new location of the origin outside the three cones is computed and the three contact points together with their friction cones are translated accordingly. Discussion about this computation will be given in Section V. Once the three dual line segments and their signs of moment are obtained, Proposition 3 can then be directly applied. The convex hull of two line segments is the convex hull of the four endpoints, so the condition (b) of Proposition 3 amounts to detecting intersection between a line segment and a convex polygon of at most four vertices. Note that computing the convex hull of points in the plane and detecting intersection between a line segment and a convex polygon are basic problems in computational geometry. Efficient algorithms for solving these problems can be found in [2].

\section{LOCATING THE ORIGIN}

To map a double-sided cone to the corresponding dual line segment, Lemma 2 requires that the cone does not contain the origin of the primary plane. The containment can be easily detected by comparing the half cone angle with the angle between each cone's axis and the line joining the origin to the cone's apex. Having the angle greater than the half cone angle indicates that the origin is outside the cone. As mentioned in Section IV-C, some configurations may violate the assumption of Lemma 2 by having the origin contained in some friction cones. In this case, a new location of the origin outside the three double-sided friction cones has to be located. A straightforward method is to pick a point outside the union of the three cones. Although computing the union of three double sided cones can be solved efficiently using algorithms from computational geometry for obtaining the union of polygons [2], we sketch here a much simpler and efficient method for finding a point outside three double sided cones.

The method is based on an intuitive idea that a line cutting through each of the three cones (i.e., separating each cone into two pieces) must contain a point outside the union of the three cones (Fig. 7(a)). To find such a line, notice that each cone has an interval of orientations of all lines it entirely contains. A line with an orientation outside the union of the three intervals from the three cones definitely cut through all the three cones (Fig. 7(b)). This is because it has a different orientation from every line in each cone. We choose a line with this orientation that passes through a cone's apex so that only four line intersection has to be computed in identifying segments of the line that are covered by the cones. Any point on the line outside these segments can be chosen to be a new origin (Fig. 7(c)).

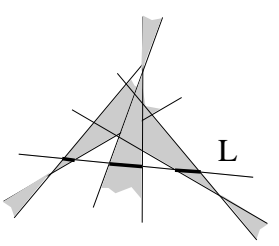

(a)

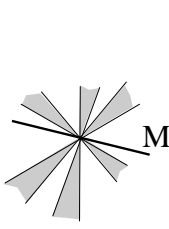

(b)

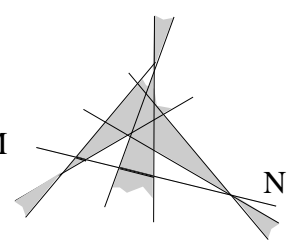

(c)
Fig. 7. Picking a new origin: (a) line $L$ cutting through the three cones contains points outside the cones, (b) line $M$ has an orientation outside the union of the three intervals of orientations from the three cones, and (c) line $N$ parallel to $M$ and through an apex of a cone allowing us to identify only two segments covered by the cones

Using the above method, a point outside the three cones clearly can be found when the union of the three orientation intervals does not cover the entire orientation range $\mathcal{S}^{1}$. This means that the method can always find a new origin outside the three cones when the half friction cone angle is smaller than $\frac{\pi}{6}$ (so that the union of the three intervals is smaller than $2 \pi$ ). Although this method has a limitation, it is quite practical since the half friction cone angle smaller than $\frac{\pi}{6}$ is usually assumed in most works on grasping.

An interesting alternative to the workaround method given in this section is to allow friction cones to contain the origin. It is easy to verify that the dual of all forces in a friction cone that contains the origin is, of course, not a line segment but the union of two rays lying on the same line with different signs of moment. This approach requires a direct modification of Lemma 2, a minor change in Proposition 3, and a new algorithm that can handle rays. We are completing this modification and in the process of writing a paper describing this new approach. 


\section{CONCLUSION}

We have presented a new necessary and sufficient condition for three hard fingers with frictional contact to form a forceclosure grasp in two dimensions. Following the new condition, we have transformed force-closure test into the problem of detecting intersection between a line segment and a convex polygon of at most four vertices. Besides an efficient forceclosure test, an important contribution of this work is the dual representation of friction cones. Hopefully this representation will help open a new way to look at grasping problems particularly the ones involving force-closure. Besides extending the approach to cover the cases where friction cones may contain the origin, we are investigating the dual representation in many aspects. An interesting avenue is an attempt in rewriting everything using projective geometry in hope that rays and segments may be treated uniformly. We are also exploring how to apply this new representation in solving grasp planning and regrasping problems.

\section{REFERENCES}

[1] A. Bicchi. On the closure properties of robotics grasping. International Journal of Robotics Research, 14(4):319-334, 1995.

[2] M. de Berg, M. van Kreveld, M. Overmars, and O. Schwarzkopf. Computational Geometry: Algorithms and Applications. Springer, 1997.

[3] D. Ding, Y-H Liu, and S. Wang. Computation of 3-d form-closure grasps IEEE Transactions on Robotics and Automation, 17(4):515-522, 2001.

[4] J-W. Li, H. Liu, and H-G. Cai. On computing three-finger force-closure grasps of 2-d and 3-d objects. IEEE Transactions on Robotics and Automation, 19(1):155-161, 2003.

[5] V-D. Nguyen. Constructing force-closure grasps. International Journal of Robotics Research, 7(3):3-16, June 1988.

[6] J. Ponce and B. Faverjon. On computing three-finger force-closure grasps of polygonal objects. IEEE Transactions on Robotics and Automation, 11(6):868-881, December 1995.

[7] J.K. Salisbury and B. Roth. Kinematic and force analysis of articulated hands. ASME Journal of Mechanisms, Transmissions, and Automation in Design, 105:33-41, 1982. 